\title{
How do you perform your tympanoplasty, endoscopically or microscopically?
}

\author{
Ali Bayram¹, MD - Daniele Marchioni², MD - Kevin Peng ${ }^{3}$, MD - II Joon Moon ${ }^{4}$, MD - Cemal Cingi ${ }^{5}$, MD \\ 1: Department of Otolaryngology, Kayseri City Hospital, Kayseri, Turkey, ORCID: 0000-0002-0061-1755 \\ 2: Department of Otolaryngology, University Hospital of Verona, Verona, Italy, ORCID: 0000-0002-8004-7284 \\ 3: Department of Otolaryngology, House Clinic, Los Angeles, California, United States, ORCID: 0000-0002-9166-0869 \\ 4: Department of Otolaryngology, Samsung Medical Center, Sungkyunkwan University School of Medicine, Seoul, Korea \\ ORCID: 0000-0002-3613-0734 \\ 5: Department of Otolaryngology, University of Osmangazi, Eskişehir, Turkey, ORCID: 0000-0003-3934-5092
}

\begin{abstract}
Since the introduction of endoscopic ear surgery (EES) in the 1990s, endoscopic systems have been utilized in a number of ear surgeries including myringoplasty and tympanoplasty. Endoscopic tympanoplasty (ET) is described as a minimally invasive surgery for surgical repair of the tympanic membrane (TM) with comparable graft survival and hearing outcomes to conventional microscopic surgery (CMT). In the present review, we aimed to outline the surgical benefits and potential drawbacks of ET with clinical outcomes in order to determine whether ET constitutes a proper alternative to CMT.
\end{abstract}

Keywords: Tympanic membrane perforation, tympanoplasty, myringoplasty, endoscopes.

\section{Introduction}

Tympanic membrane (TM) perforation is a common pathological condition that occurs due to various etiologies including infection, trauma or idiopathic causes. ${ }^{[1]}$ Repair of TM perforation can be achieved by tympanoplasty or myringoplasty, which are differentiated according to the pathological involvement of the TM and middle ear. The tympanoplasty procedure involves closure of the TM perforation in conjunction with management of chronic otitis media, cholesteatoma and hearing restoration. Conventional microscopic tympanoplasty (CMT) has been the standard method for surgical repair of the TM worldwide with a success rate of $80-90 \% .{ }^{[2]}$ CMT can be performed via transcanal, endaural or postauricular routes with a variety of graft material, for which temporalis fascia and perichondrium have been mostly used. Among these routes, the postauricular approach with a microscope offers significant advantages to the surgeon by enhancing the visibility of the operative site, particularly for large or anterior TM perforations, as well as for patients with anterior canal wall bulge. ${ }^{[3]}$ However, a postauricular approach usually necessitates hair shaving, incision and soft tissue dissection, as well as general anesthesia. Also, the surgical view during microscopic sur- 
gery is usually affected by the width, tortuosity and bony overhangs of the ear canal, therefore canalplasty is often performed to improve the vision. Recently, endoscopic systems have gained popularity as a single operative tool or as an assistive tool in a microscopic approach for otological surgery. In 1978, Eichner ${ }^{[4]}$ introduced endoscopic systems for otologic examination with a $2.7 \mathrm{~mm}$ diameter rigid endoscope. Since its first utilization for ear surgery in the late 1990s, endoscopic systems have promised better vision of the deep and hidden parts of the middle ear cavity, such as anterior and posterior epitympanic spaces, eustachian tube, facial recess, sinus tympani and hypotympanum (Figure 1). ${ }^{[5]}$ With accumulated experience since 1990, a number of ear surgeries can be performed via an endoscopic approach, including ventilation tube insertion, repair of TM perforation, ossicular reconstruction, otosclerosis and cholesteatoma surgery and cochlear implantation.

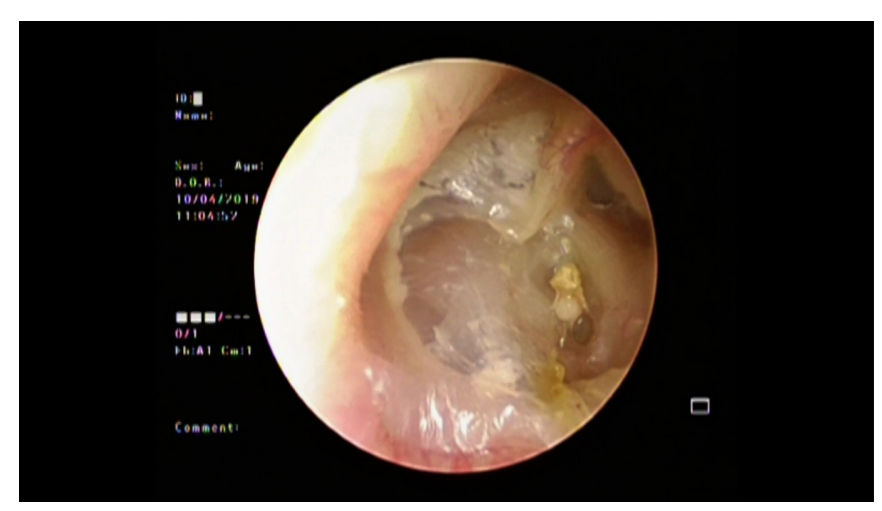

Figure 1. Endoscopic view of adhesive tympanic membrane with an eroded long arm of the incus.

As in other highly sophisticated surgical procedures, endoscopic ear surgery (EES) necessitates special instruments, as implied by the nomenclature of the procedure. The $0^{\circ}, 30^{\circ}$ and $45^{\circ}$ angled rigid endoscopes $2.7 \mathrm{~mm}, 3$ $\mathrm{mm}$ and $4 \mathrm{~mm}$ in diameter with working lengths of $6 \mathrm{~cm}$, $11 \mathrm{~cm}$ and $18 \mathrm{~cm}$ are the most commonly used endoscope types during EES. ${ }^{[6]}$ A high-resolution camera with a monitor located opposite to the surgeon, light source and fiber optic cable are also required. The International Working Group on Endoscopic Ear Surgery (IWGEES) has been working on the development of special equipment and instruments for EES based on more than 15 years of experience. ${ }^{[6]}$ In recent years, one of the most important develop- ments in endoscopic instruments is incorporating suction into the instrument shaft, hence enabling the removal of blood and debris from the surgical field. Although these instruments provide the ability to perform dissection and aspiration maneuvers simultaneously, the main disadvantage is frequent occlusion during suctioning. During EES, a surgical microscope should still be available for switching to microscopic surgery in the surgical setting.

The endoscopic tympanoplasty (ET) procedure initiates with placement of the patient in an otosurgical position. The distance between the shoulder and pinna should be increased in order to improve ergonomics. The surgical procedure is mostly performed through $0^{\circ}$ or $30^{\circ}, 3 \times 11$ to $14-\mathrm{cm}$ rigid endoscopes. A $45^{\circ}$ angled rigid endoscope is usually recommended during evaluation of the protympanum, retrotympanum and ventilation routes. Optimal hemostasis is essential during ET and is achieved by infiltration of local anesthetic and vasoconstrictor agents into the ear canal and graft donor site, placement of 1:2000 epinephrine soaked cottonoids over the TM remnant for 10 minutes and total intravenous anesthesia with a targeted mean blood pressure of $60 \mathrm{~mm} \mathrm{Hg}$ and heart rate of 60 $\mathrm{bpm}$. After arranging the optimal circumstances for hemostasis, a graft material such as tragal perichondrium and cartilage, temporalis muscle fascia or fat tissue is harvested. Then the edges of the TM perforations are denuded and the approach to the middle ear is performed either by endocanal (without tympanomeatal flap) or transcanal technique. During the transcanal, underlay technique, a wide front tympanomeatal flap is elevated and the posterior annulus is identified and lifted from the sulcus to provide access into the middle ear. Middle ear ossicles are examined for integrity and movement, and reconstruction of the ossicles is performed when necessary. Permeability of the anterior and posterior isthmus is checked, and graft material is positioned and supported by the placement of sponges (Figure 2-5). ${ }^{[1]}$

During conventional microscopic cholesteatoma surgery, the benefit of endoscopic assistance for removing residual disease has been demonstrated in the literature. ${ }^{[7-9]}$ In recent studies, ET has also been demonstrated with favorable results as a minimally invasive surgery. ${ }^{[10-12]}$ ET enables a wider view with varying angles of view, and does not require canalplasty, postauricular incision and general anesthesia; hence, the operative time is shorter than with conventional techniques. The minimally invasive nature of 


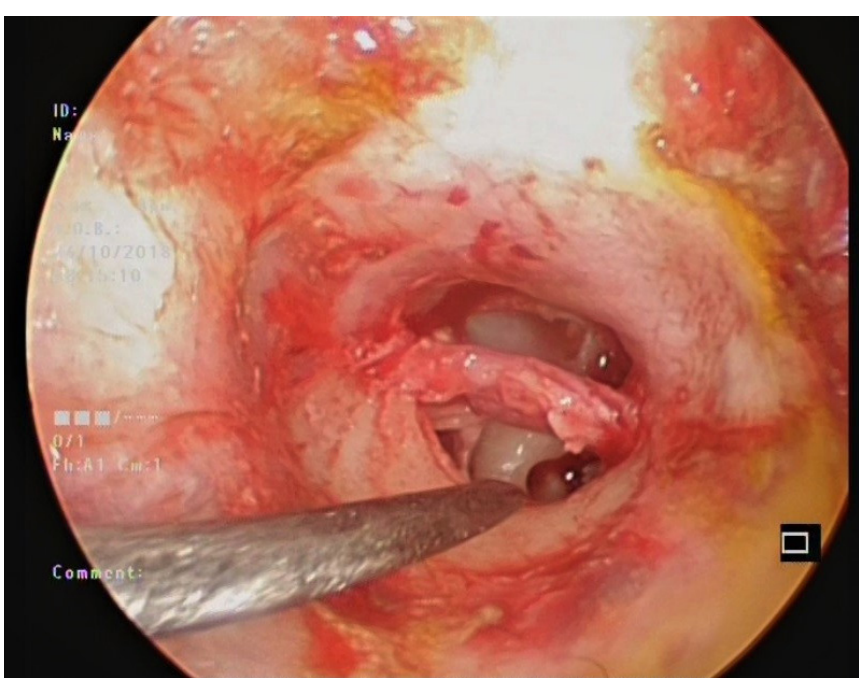

Figure 2. Endoscopic transcanal tympanomeatal flap elevation and access to the middle ear.

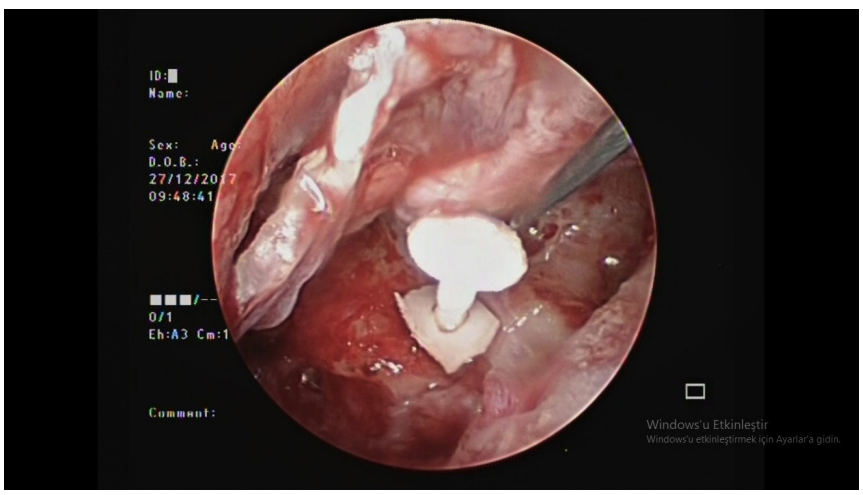

Figure 4. Endoscopic tympanoplasty with total ossicular replacement prosthesis (TORP) placement.

ET ensures better cosmetic outcomes with less postoperative pain and morbidity. Endoscopic systems with different angles enable observation of deep and hidden areas of the middle ear cavity such as the facial recess, sinus tympani, anterior attic, anterior mesotympanum, eustachian tube and hypotympanium, hence allowing detection of the origin of any selective atelectasis or poor ventilation during the ear surgery. ${ }^{[1,7]}$ Although endoscopic approaches bring substantial advantages during tympanoplasty, they have a number of disadvantages such as one-handed surgery, difficulty in depth perception, deterioration of endoscopic view due to blood or fog and the risk of direct or thermal injury. ${ }^{[13,14]}$ Therefore, in this review, we aimed to outline the sur-

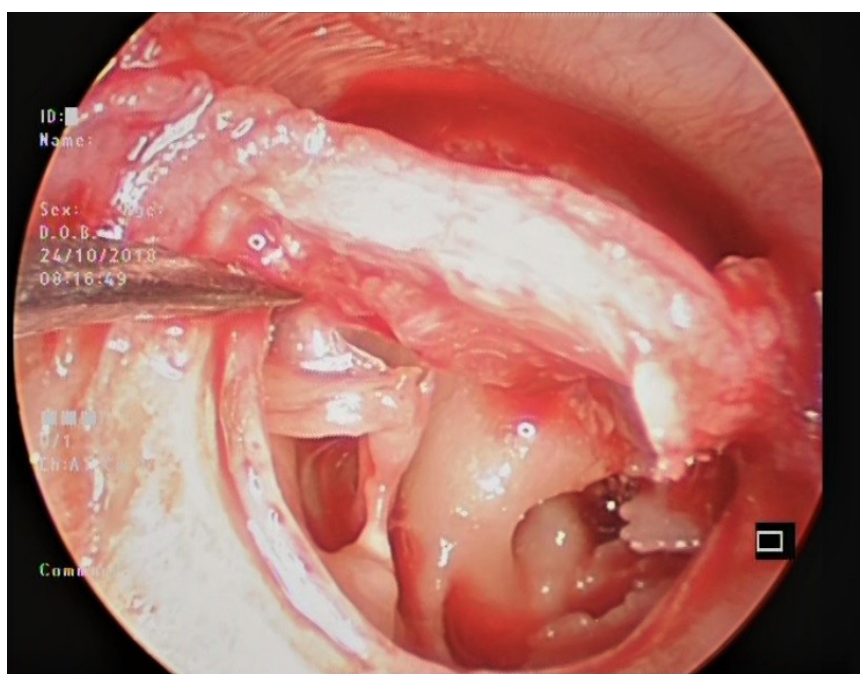

Figure 3. Endoscopic view of incudostapedial joint, tympanic segment of the facial nerve, stapes, stapedial tendon, eminentia pyramidalis, and round window.

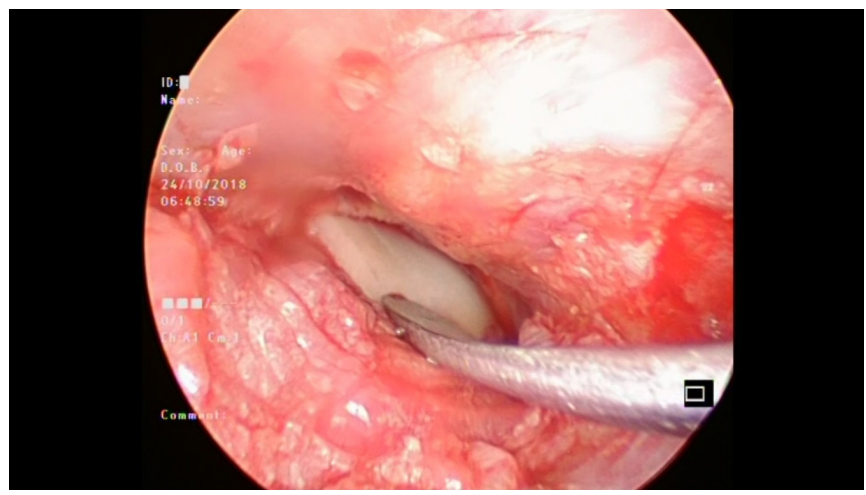

Figure 5. Endoscopic closure of tympanic membrane perforation with cartilage graft.

gical benefits and potential drawbacks of ET with clinical outcomes in order to determine whether ET constitutes a proper alternative to CMT.

\section{Discussion}

Although microscopic ear surgery (MES) offers many advantages to surgeons, such as binocular vision with magnified surgical area and the possibility of two-handed surgery, clinicians require newer surgical trends and sophisticated technologies that enable the visualization of deep and hidden areas of the middle ear cavity with the consideration of minimal invasiveness. Conventional MES usually necessitates soft tissue dissection and bone drilling 
to improve visualization, which frequently means more surgical work, longer operative time and a more extensive healing process. ${ }^{[13]}$ However, MES may sometimes still fall short to the surgeon's requirements, despite maneuvers performed to achieve better surgical visualization. Therefore, attempts to involve endoscopic systems in ear surgery initially aimed to respond to this need. As surgeons have realized the advantages of this new surgical trend, the utilization of endoscopic systems has been expanded and has evolved into more advanced ear surgeries. Concordantly, modern tympanoplasty techniques have also taken their share from recent technological advances, and presently, ET promises ear surgeons a clear and high-definition surgical view in patients with a narrower ear canal or anterior TM perforations by means of angled lenses and powerful light sources positioned at the tip of the endoscope with no external incision and less surgical dissection. On the other hand, although conventional MES is still favored by most surgeons as a standard surgical method during tympanoplasty or myringoplasty, there are a number of studies aimed to compare surgical facilities and clinical outcomes of ET versus CMT. ${ }^{[1,3,10,11]}$

Two systematic reviews revealed that graft survival rate and hearing outcomes following ET were comparable with CMT. ${ }^{[12,15]}$ In the qualitative analysis of Lee et al ${ }^{[15]}$, the graft survival rate in ET ranged from $83 \%$ to $100 \%$, which was similar to that in CMT according to the studies included in their meta-analysis. The graft survival rate seems to be affected by the grafting technique rather than the type of surgical approach, although most of the studies regarding the outcomes of ET utilized an underlay grafting technique during ET, which may constitute a potential publication bias ${ }^{[15]}$. In the review of Tseng et al ${ }^{[12]}$, the comparable improvement rate of air-bone gaps of ET and CMT was attributed to the similar TM closure rates for both ET and CMT, although none of the included studies were fully compliant with American Academy of Otolaryngology - Head and Neck Surgery reporting guidelines. ${ }^{[16]}$ Lee et al ${ }^{[15]}$ also reported a comparable hearing improvement of ET and CMT in their systematic review. However, pooled analysis of their study demonstrated greater hearing improvement in ET than CMT, which was likely secondary to several factors. The authors addressed these factors as disadvantages of endoscopic surgery such as one-handed technique, potential publication bias and a lack of subgroup analysis performed according to patient characteristics including perforation site or size and revision surgery.
The operative time was reported to be shorter for ET than CMT for both children and adults. ${ }^{[11,17]}$ Kuo and Wu ${ }^{[11]}$ found the operative time was significantly shorter for 74 ears that underwent ET than the 57 ears that had CMT. Patel et al reported that the mean operative time for ET and CMT was $75 \mathrm{~min}$ and $90 \mathrm{~min}$, respectively. ${ }^{[18]}$ These results may not be surprising since ET necessitates less management of soft tissue and bony structures to provide adequate visualization. Nevertheless, there are some issues that have to be addressed that lengthen the duration of the ET procedure, such as long learning curve for being familiar with endoscopic technique in ear surgery and the obligation of wiping the fogged or blood-stained endoscope several times during the surgery. Kuo et al indicated that more practice and a higher learning curve are needed for shortening the time of ET even for surgeons experienced in MES. ${ }^{[11]}$

During MES, the annulus ring may not be fully visualized in nearly $17-20 \%$ of patients due to a stenotic ear canal or bony protrusions in MES. In such a condition, surgeons usually are required to perform a canalplasty for drilling out the protruded bone. ${ }^{[15,19,20]}$ By contrast, the canalplasty rate was reported to be significantly lower in ET than in CMT since the endoscopy offers wider and all-round surgical view to the surgeons. ET also does not necessitate postauricular incision, which means better postoperative cosmetic results including no postauricular scar, auricular deformity or pinna asymmetry. ${ }^{[3,12,21]} \mathrm{CMT}$ requires more postoperative care such as a compression bandage for a postauricular incision, which causes a later return to routine daily activity than in ET. ${ }^{[22]}$ Postoperative pain and complications such as wound dehiscence or infection were reported to be significantly lower in ET than CMT. ${ }^{[23,24]}$ Choi et al ${ }^{[3]}$ used a sequential postoperative pain scale (NRS-11) for patients who had ET and CMT and demonstrated that the pain scale score was significantly lower in the ET group one day after surgery. Tseng et al ${ }^{[25]}$ evaluated subjective outcomes of patients who had an endoscopic transcanal myringoplasty, including pain, duration of pain medication and days required to resume routine activities and showed favorable short-term subjective outcomes. In a prospective randomized controlled trial consisting of 13 patients who had ET in one ear and CMT on the contralateral side, Kaya et al ${ }^{[26]}$ reported shorter surgery time, better health status and lower pain with comparable improvement in air-bone gap and graft success in ET. ET can be performed as an outpatient surgery due to its minimally 
invasive characteristic, and thus medical expenditure is decreased if no major postoperative complications occur after the procedure. ${ }^{[1]}$

Anterior TM perforations are an important cause of graft failure in surgical repair of the TM. Nardone et al ${ }^{[27]}$ showed that anterior perforations had a negative impact on the operative success of myringoplasties in their study including 1040 cases. Anterior TM perforations may necessitate a postauricular approach or canalplasty for entire vision of the perforation with additional surgical morbidity. An endoscopic transcanal surgery is suggested to provide adequate vision without a postauricular approach or canalplasty with comparable success rates in anterior TM perforations. ${ }^{[28-30]}$ Eren et al ${ }^{[28]}$ reported a $95.5 \%$ closure rate for anterior TM perforation with an improved airbone gap from $19.3 \pm 3.3 \mathrm{~dB}$ to $10.8 \pm 4.5 \mathrm{~dB}$ in 21 of $22 \mathrm{pa}-$ tients who underwent a cartilage butterfly myringoplasty with an endoscopic transcanal approach. El-Hennawi et al [30] compared endoscopic push-through myringoplasty (28 patients) and microscopic myringoplasty (28 patients) with a tragal cartilage perichondrial graft and reported that the endoscopic technique is as effective as a microscopic approach in terms of surgical success rates with less operative time and invasiveness. In their study, none of the patients had a canalplasty in the endoscopic group while canalplasty was performed for 10 of the 28 patients in the microscopic group. Plodpai ${ }^{[31]}$ performed a similar comparison for large TM perforations with endoscopic or microscopic overlay techniques. The author found the graft survival rate to be comparable with both techniques, whereas improvement of the air-bone gap was significantly better in the endoscopic group with less invasiveness.

Although the endoscopic approach provides considerable advantages in ear surgery including tympanoplasty or myringoplasty, it has some disadvantages that need to be addressed. The necessity of handling endoscopes hinders two-handed surgery and limits the surgeon's maneuvers for simultaneous blood suction and tissue removal. Endoscopic systems provide two-dimensional views instead of

\section{References}

1. Anzola JF, Nogueira JF. Endoscopic Techniques in Tympanoplasty. Otolaryngol Clin North Am 2016;49:1253-64.

2. Dornhoffer JL. Hearing results with cartilage tympanoplasty. Laryngoscope 1997;107:1094-9.

3. Choi N, Noh Y, Park W, et al. Comparison of Endoscopic Tympanoplasty to Microscopic Tympanoplasty. Clin Exp Otorhinolaryngol 2017;10:44-9. three-dimensional images obtained from binocular microscopic systems. Therefore, the endoscopic approach carries potential risks for trauma to the surrounding structures due to the difficulty in depth perception of the surgeons and the one-handed surgical technique. The ossicular chain may suffer because of the excessive manipulation or direct trauma of the tip of the endoscopes during the EES. Nevertheless, Marchioni ${ }^{[32]}$ reported only one case of accidental disjunction of the ossicular chain in 825 patients who underwent EES. Especially under local anesthesia, surgeons must be cautious regarding a patient's unintentional head movements. ${ }^{[33]}$ Also, potential thermal injury generated by the light source of endoscopic systems should be taken into account, and can be remedied by adjusting the light to the lowest power level that will still allow for adequate visualization. ${ }^{[34,35]}$ Ozturan et al ${ }^{[36]}$ studied intraoperative thermal safety in 12 patients who underwent myringoplasty and reported that intermittent irrigation or frequent aspiration may prevent thermal injury with light intensity settings of $50 \%$. Also, xenon light sources have a very high light intensity; however, light emitting diode (LED) light sources may provide adequate illumination with low intensity settings and at cooler temperatures.

\section{Conclusion}

Based on the growing experience since the late 1990s, endoscopic systems have been utilized in a number of ear surgeries including myringoplasty and tympanoplasty. Besides providing comparable results regarding graft survival and hearing outcomes as conventional microscopic surgery, ET also promises lower surgical morbidity and better cosmetic results, which serves the philosophy of minimally invasive surgery. However, a steeper learning curve, necessity of special instrumentation and potential drawbacks of the surgical technique such as one-handed surgery and difficulty in depth perception should be taken into account by surgeons, with no hesitation switching to microscopic surgery whenever needed.
4. Eichner H. A "mother-and baby scope-optic" for middle ear endoscopy. Laryngol Rhinol Otol (Stuttg) 1978;57:872-6.

5. Thomassin JM, Duchon-Doris JM, Emram B, Rud C, Conciatori J, Vilcoq P. Endoscopic ear surgery: initial evaluation. Ann Otolaryngol Chir Cervicofac 1990;107:564-70.

6. Badr-El-Dine M, James AL, Panetti G, Marchioni D, Presutti L, Nogue- 
ira JF. Instrumentation and technologies in endoscopic ear surgery. Otolaryngol Clin North Am 2013;46:211-25.

7. Tarabichi M, Nogueira JF, Marchioni D, Presutti L, Pothier DD, Ayache S. Transcanal endoscopic management of cholesteatoma. Otolaryngol Clin North Am 2013;46:107-30.

8. Badr-el-Dine M. Value of ear endoscopy in cholesteatoma surgery. Otol Neurotol 2002;23:631-5.

9. Good GM, Isaacson G. Otoendoscopy for improved pediatric cholesteatoma removal. Ann Otol Rhinol Laryngol 1999;108:893-6.

10. Ohki M, Kikuchi S, Tanaka S. Endoscopic Type 1 Tympanoplasty in Chronic Otitis Media: Comparative Study with a Postauricular Microscopic Approach. Otolaryngol Head Neck Surg 2019 Mar 26:194599819838778. doi: 10.1177/0194599819838778 [Epub ahead of print]

11. Kuo CH, Wu HM. Comparison of endoscopic and microscopic tympanoplasty. Eur Arch Otorhinolaryngol 2017;274:2727-32.

12. Tseng CC, Lai MT, Wu CC, Yuan SP, Ding YF. Comparison of the efficacy of endoscopic tympanoplasty and microscopic tympanoplasty: A systematic review and meta-analysis. Laryngoscope 2017;127:1890-6.

13. Kozin ED, Gulati S, Kaplan AB, et al. Systematic review of outcomes following observational and operative endoscopic middle ear surgery. Laryngoscope 2015;125:1205-14.

14. Bottrill I, Perrault DF Jr, Poe D. In vitro and in vivo determination of the thermal effect of middle ear endoscopy. Laryngoscope 1996;106:213-6.

15. Lee SY, Lee DY, Seo Y, Kim YH. Can Endoscopic Tympanoplasty Be a Good Alternative to Microscopic Tympanoplasty? A Systematic Review and Meta-Analysis. Clin Exp Otorhinolaryngol 2019;12:145-55.

16. Committee on hearing and equilibrium guidelines for the evaluation results of treatment of conductive hearing loss. American Academy of Otolaryngology-Head and Neck Surgery Foundation, Inc. Otolaryngol Head Neck Surg 1995;113:186-7.

17. Dündar R, Kulduk E, Soy FK, et al. Endoscopic versus microscopic approach to type 1 tympanoplasty in children. Int J Pediatr Otorhinolaryngol 2014;78:1084-9.

18. Patel J, Aiyer RG, Gajjar Y, Gupta R, Raval J, Suthar PP. Endoscopic tympanoplasty vs microscopic tympanoplasty in tubotympanic CSOM: a comparative study of 44 cases. Int J Res Med Sci 2015;3:1953-7.

19. Glasscock ME, Jackson CG, Nissen AJ, Schwaber MK. Postauricular undersurface tympanic membrane grafting: a follow-up report. Laryngoscope 1982;92:718-27.

20. Sheehy JL, Anderson RG. Myringoplasty: a review of 472 cases. Ann Oto Rhinol Laryngol 1980;89:331-4.

21. Akyigit A, Sakallıglu O, Karlidag T. Endoscopic tympanoplasty. J Otol 2017;12:62-7.
22. Harugop AS, Mudhol RS, Godhi RA. A comparative study of endoscope assisted myringoplasty. Indian J Otolaryngol Head Neck Surg 2008;60:298-302

23. Plodpai Y, Paje N. The outcomes of overlay myringoplasty: endoscopic versus microscopic approach. Am J Otolaryngol 2017;38:542-6.

24. Kumar M, Kanaujia SK, Singh A. A comparative study of endoscopic myringoplasty vs conventional myringoplasty. Int J Otorhinolaryngol Clin 2015;7:132-7.

25. Tseng CC, Lai MT, Wu CC, Yuan SP, Ding YF. Short-term Subjective and Objective Outcomes of Patients Receiving Endoscopic Transcanal Myringoplasty for Repairing Tympanic Perforations. Otolaryngol Head Neck Surg 2018;158:337-42.

26. Kaya I, Sezgin B, Sergin D, et al. Endoscopic versus microscopic type 1 tympanoplasty in the same patients: a prospective randomized controlled trial. Eur Arch Otorhinolaryngol 2017;274:3343-9.

27. Nardone M, Sommerville R, Bowman J, Danesi G. Myringoplasty in simple chronic otitis media: critical analysis of long-term results in a 1,000-adult patient series. Otol Neurotol 2012;33:48-53.

28. Eren SB, Tugrul S, Ozucer B, Veyseller B, Aksoy F, Ozturan O. Endoscopic Transcanal Inlay Myringoplasty: Alternative Approach for Anterior Perforations. Otolaryngol Head Neck Surg 2015;153:891-3.

29. Tseng CC, Lai MT, Wu CC, Yuan SP, Ding YF. Endoscopic Transcanal Myringoplasty for Anterior Perforations of the Tympanic Membrane. JAMA Otolaryngol Head Neck Surg 2016;142:1088-93.

30. El-Hennawi DEM, Ahmed MR, Abou-Halawa AS, Al-Hamtary MA. Endoscopic push-through technique compared to microscopic underlay myringoplasty in anterior tympanic membrane perforations. J Laryngol Otol 2018;132:509-13.

31. Plodpai Y. Endoscopic vs Microscopic Overlay Tympanoplasty for Correcting Large Tympanic Membrane Perforations: A Randomized Clinical Trial. Otolaryngol Head Neck Surg 2018;159:879-86.

32. Marchioni D, Rubini A, Gazzini L, et al. Complications in Endoscopic Ear Surgery. Otol Neurotol 2018;39:1012-7.

33. Raj A, Meher R. Endoscopic transcanal myringoplasty-a study. Indian J Otolaryngol 2001;53:47-9.

34. Yung MW. The use of middle ear endoscopy: has residual cholesteatoma been eliminated? J Laryngol Otol 2001;115:958-61.

35. Rosenberg SI, Silverstein H, Willcox TO, Gordon MA. Endoscopy in otology and neurotology. Am J Otol 1994;15:168-72.

36. Ozturan O, Dogan R, Eren SB, Aksoy F. Intraoperative thermal safety of endoscopic ear surgery utilizing a holder. Am J Otolaryngol 2018;39:58591.

This is an open access article distributed under the terms of the Creative Commons Attribution-NonCommercial-NoDerivs 3.0 Unported (CC BY- NC-ND3.0) Licence (http://creativecommons.org/licenses/by-nc-nd/3.0/) which permits unrestricted noncommercial use, distribution, and reproduc- tion in any medium, provided the original work is properly cited.

Please cite this article as: Bayram A, Marchioni D, Peng K, Moon IJ, Cingi C. How do you perform your tympanoplasty, endoscopically or microscopically? ENT Updates 2019;9(2): 144-149. 